\title{
Global precedence as a postperceptual effect: An analysis of speed-accuracy tradeoff functions
}

\author{
LOUIS C. BOER and P. J. G. KEUSS \\ Free University, 1007 MC Amsterdam, The Netherlands
}

\begin{abstract}
Two experiments examined the speed-accuracy tradeoff for stimuli used by Martin (1979), some of which have a Stroop-like conflict between the relevant (to-be-judged) and the irrelevant aspect. Speed of transmitting information about a local aspect was significantly reduced when the irrelevant global aspect conflicted with the relevant local aspect, while speed of transmitting information about the global aspect was not affected when the irrelevant local aspect conflicted with the relevant global aspect. This result, when extrapolated to the accuracy level of an ordinary reaction-time task, fitted very well the reaction-time predictions of the global precedence model proposed by Navon (1977). However, other results were incongruent with the fundamental assumption of that model: that global features are accumulated with temporal priority over local features. The finding that, independently of speed, information transmission of the global aspect started later when the irrelevant local aspect was conflicting, corroborates Miller's (1981a) conclusion that global and local features are available with a similar time course. Global precedence is therefore a postperceptual effect; absence of interaction with S-R compatibility suggested that it operated before the response selection stage. The term global dominance may be preferred, because it avoids the implication of prior availability for the global aspect. Furthermore, the possibility of whether Stroop conflict should be considered a necessary condition for global dominance is discussed.
\end{abstract}

This article is concerned with the accumulation of information of a visual form and, in particular, with whether transmission of global information precedes transmission of local (detail) information. For the purpose of this paper, we conceive of perception as a process of gradual feature accumulation. Furthermore, in line with the continuous-flow model of Eriksen and Schultz $(1978,1979)$, we assume that a subject's response is based upon the amount of information accumulated at that particular instant. The ontogenesis of the percept ("Aktualgenese" in terms of the Leipzig School of Gestalt Psychology) will therefore be reflected by the response of the moment.

For the global-to-local hypothesis, the distinction between global and local features is a basic one. As opposed to feature models in which global features are composites of local features (Gibson, 1969; Selfridge, 1959; Treisman \& Gelade, 1980), the globalto-local hypothesis states that perception begins with a global whole to which local details are gradually added. According to the global precedence model, global and local features denote separate sets of features. Thus, global feature availability is not depen-

Address reprint requests to L. C. Boer, who is presently at the Institute for Perception TNO, P.O. Box 23, 3769 ZG Soesterberg, The Netherlands. The second author's address is: Department of Psychology, Free University, Provisorium I, P.O. Box 7161, 1007 MC Amsterdam, The Netherlands. The authors would like to acknowledge their indebtedness to the Institute for Perception for the use of its laboratory and for the time made available to the first author. dent on the accumulation of a sufficient number of local features; on the contrary, the model implies that global feature sampling precedes local feature sampling or that global feature sampling is faster than local feature sampling. Some predictions from the global precedence model are: (1) it is easier to judge a form on its global characteristics than on its local characteristics; and (2) global characteristics are difficult to ignore, while local ones are easy to ignore (that is, interference from irrelevant (to-beignored) global aspects exceeds interference from irrelevant local aspects).

What is meant by the global-local distinction is rather obvious in everyday language. A more formal definition can be attempted using the concept of spatial frequency. A visual form can be described as a mixture of different spatial frequencies (Campbell, 1974). A way of eliminating high spatial frequencies is to defocus the image, as, for example, the image from a projector can easily be defocused. Local or detailed information contained in the high frequencies is lost, while gross, global shapes contained in the low frequencies are preserved. High spatial frequencies in isolation (i.e., without low frequencies) are exemplified by a bad quality Xerox copy. Details are preserved, but global, uniform surfaces seem to have brightness differences. Another formal definition assumes that the outer contour is the global aspect; Bouma (1971, p. 463) called this contour, formed by the smallest polygon enclosing the whole, the "envelope." Which 
definition is more useful is not clear. Moreover, the choice of one definition over the other does not seem to be of consequence for the research of this paper.

One line of evidence for the global precedence model is obtained from the analysis of confusion matrices. Frequently, briefly flashed letters and letterlike symbols are confused with each other if similar in global characteristics, as defined by contour similarity (Bouma, 1971; Lupker, 1979). Of special interest for the present study is another line of evidence that focuses on Stroop interference in letter stimuli. The interference is between global shape and the elements making up that shape. For example, $\mathrm{H}$ and $\mathrm{S}$ are presented as global shapes in Figure 1, in which the component elements are either small capital Hs or Ss (or Os). When the response set consists of $\mathrm{H}$ and $\mathrm{S}$, an $\mathrm{H}$ consisting of many small $\mathrm{Hs}$ is said to be consistent, while an $\mathrm{H}$ consisting of small Ss is said to be conflicting (as is an $\mathrm{S}$ consisting of small Hs). The typical task requires observers to classify according to a particular level and to ignore the other level as much as possible. It follows that a performance difference between consistent and conflict stimuli indicates a failure to ignore the irrelevant level.

Navon $(1977,1981 a)$ found clear support for the global precedence model. Irrelevant local letters were easily ignored, while global shape was impossible to ignore. The result was replicated in part by Kinchla and Wolfe (1979) in a different paradigm. These in-
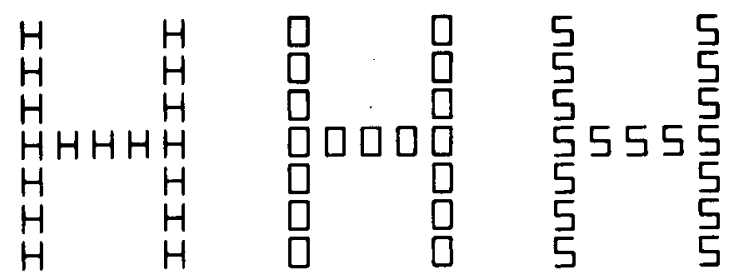

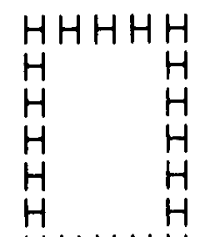

$\mathrm{HHHHH}$

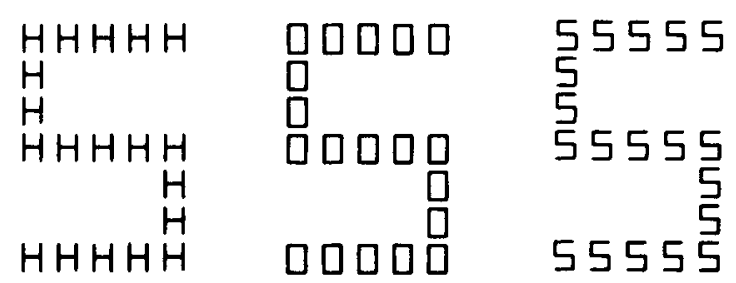

Figure 1. Global letters consisting of many local letters. (These letters were used by Martin, 1979, and in Experiment 2.) vestigators used a target search task in which the subject detected whether or not the auditory target letter was present on the display. They demonstrated global precedence provided that the large letter subsumed less than $8 \mathrm{deg}$ of visual angle. Martin (1979) also obtained similar results and showed as an additional requirement that the number of elements constituting the global shape should not be too low. Essentially, her point related to the fact that legibility of letters formed by filling in the appropriate positions of an $r \times c$ matrix is decreased with lower values of $r$ and c (Vartabedian, 1971). She found global precedence for stimuli built on a $7 \times 5$ matrix; Navon (1979, 1981a) and Kinchla and Wolfe (1979) used approximately the same matrix $(7 \times 6)$.

A study by Hoffman (1980) raises doubt about the global precedence model. Hoffman reports similar speed of recognition for "judge global" and "judge local" (attentional task instructions to subject) conditions. In addition, he reports that it is just as difficult to ignore the global level as it is to ignore the local level. It is not clear to us how this result was obtained or how it can be reconciled with other studies, especially that by Kinchla and Wolfe (1979), who used a similar paradigm. Hoffman refers tentatively to "a host of ill-defined [stimulus] factors" (p. 233). This is in line with Navon's (1981a, p. 7) argument that global precedence can be overridden by other factors. One of these factors could be the use of matrices with rather sparse points to construct the letters $(5 \times 5$ for global and $6 \times 5$ for local letters). Another factor may be due to stimulus presentation-the oscilloscope built the stimulus in such a way that the first small element was finished some $23 \mathrm{msec}$ before the last element (and, therefore, also the global shape) was finished. This difference is small, but if it had any effect it would undoubtedly tend to favor local over global aspects.' (In pilot work, we found another demonstration that global precedence can be overridden-namely, the presentation of a few of the local letters at a higher brightness. A Tektronix 611 oscilloscope constructed the stimuli of Figure 1. When the completed image was shown to the subject, the last small letter to be plotted had a 300 -mlx intensity, while the first letters to be plotted were already at the storage level of $6 \mathrm{mlx}$. It took another $75 \mathrm{msec}$ for the last element to reach storage level. The data, depicted in the left part of Figure 2, show no global precedence. When the same stimuli were presented later by a slide projector, the clear precedence result depicted in the right part of Figure 2 was obtained. ${ }^{2}$ )

In the studies considered above, reaction time (RT) is the principal dependent variable, while proportion of errors is a variable of secondary interest; an alternative method is one that combines these two variables into one measurement-namely, a speed-accuracy function (Schouten \& Bekker, 1967). According to the continuous-flow model, the initial region of the 


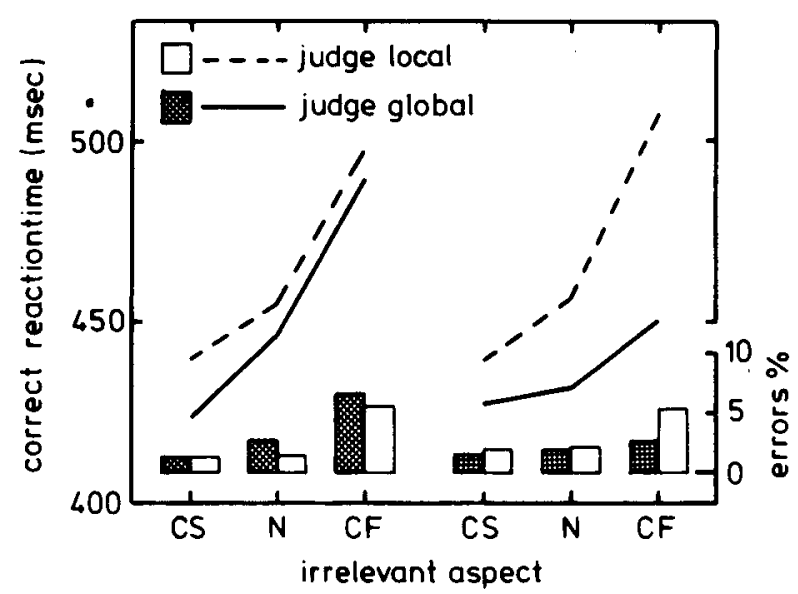

Figure 2. A demonstration that global precedence can be overridden, in this case by presenting a few local elements at a higher brightness. Both panels show RT and error rate as a function of the to-be-ignored aspect, which is consistent with (CS), neutral to (N), or in conflict with (CF) the relevant aspect. The letters depicted in Figure 1 were used as stimuli. In the left panel, they were presented by an oscilloscope, which resulted in a between-elements difference in brightness; in the right panel, they were presented by a slide projector. Note that global precedence is obtained only in the latter situation.

speed-accuracy function reflects how the subject acts on the first bits of perceptual information (Eriksen \& Schultz, 1978, p. 5). This is relevant for the global precedence model, for if global features are sampled first, they should prevail during the initial stages of perceptual processing. When the subject is forced (e.g., by imposing a deadline) to emit a very fast response, a more direct demonstration of global precedence is expected than under the usual RT instruction that warns the subject to avoid errors. In the latter case, the subject may aim at a safe response, that is, only after accumulation of sufficient global features; this increases the probability that local features are added to the sample, possibly lowering sensitivity for global precedence. Therefore, we prefer a speed-accuracy function analysis. Although in a different field, Polf (1976) also chose speed-accuracy analysis for essentially the same reason-namely, that certain representations are activated only when the subject has sufficient time.

Before proceeding with the specific predictions, some definitional framework will clarify the exposition. By the speed-accuracy function (SAF), we denote that region of the RT/accuracy curve for which RT and accuracy are positively related. The SAF describes the increases in accuracy gained by unit increases in RT. The upper bound of the function is achieved when accuracy reaches perfection; the lower bound is chance level. Expressing accuracy in bits of information transmitted between stimuli and responses (Garner, 1962), the lower bound is at the zero-bits transmitted level (i.e., when the SAF intersects the abscissa or RT axis).

Of particular interest for the SAF analysis are those stimuli for which the local aspect has some RT effect when the subject has to react to global form only. This effect, minor as it may be relative to the complementary effect (global effects when the subject reacts to local aspects only), enables us to see at which level of accuracy local features enter the percept.

In the "judge global" condition, the global precedence model predicts the following for the SAF: (1) in the initial region of the SAF, no difference between consistent and conflict stimuli is expected; (2) as accuracy approaches perfection, a decrease in the growth rate of information per time unit is expected-caused by the interference in conflict stimuli that occurs as local features enter the picture. Thus, the conflict SAF is a two-limbed function: at first, identical to the consistent SAF (since only global features are present); later, with a smaller slope, or when there is a smooth transition between the limbs, the SAF is curvilinear. The best-fitting straight line for the conflict SAF will have a shallower slope than the consistent SAF and will intersect the $\mathrm{RT}$ axis at a lower RT.

\section{EXPERIMENT 1}

The goal of the first experiment was to test the "judge global" predictions. Observers classified rectangles according to position (lying/standing). In-

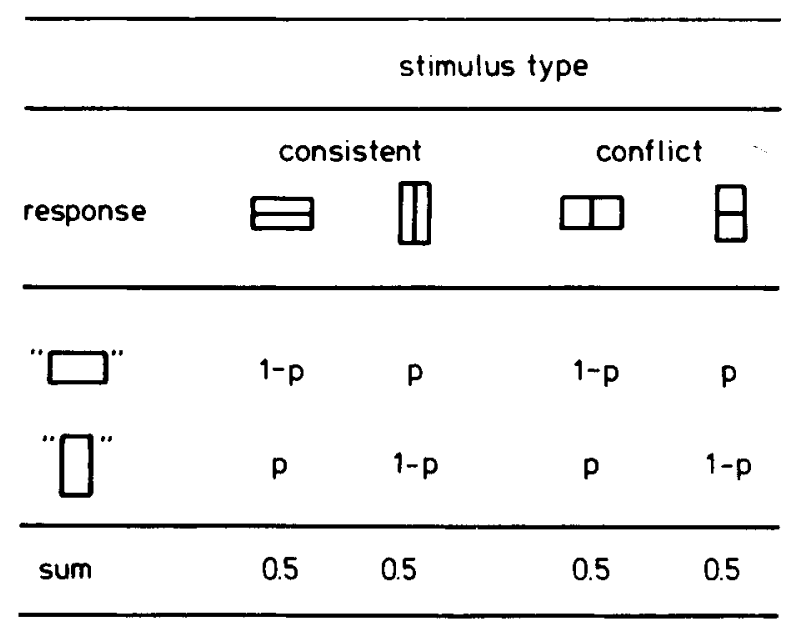

note $: P=$ Proportion of errors/stimulus

Figure 3. Stimuli used in Experiment 1 and response accuracy derivation. There are two types of stimuli: consistent and conflict. Proportions of correct and error responses at a particular deadline are entered in the two $2 \times 2$ stimulus-to-response matrices-one matrix for consistent, the other for conflict stimuli. For each deadline, within-matrix accuracy is expressed as $\mathrm{U}$ (stim:resp) (Garner, 1962). 
serted in each rectangle was a lying or standing line through the rectangle's midpoint (see Figure 3). Previous experiments (Boer \& Keuss, 1979, 1981) have shown that classifying by the rectangular outline was easier than classifying by the inserted line that is a detail in the whole. In addition, irrelevant variation in the outline was more difficult to ignore than irrelevant variation in the inserted line. All this suggested the view that the outline rectangle is a global aspect, while the inserted line is a local aspect. This view is in agreement, both with the definition that equates contour and global aspect and with the definition that equates low spatial frequency and global aspect (see Petersik, 1977, for a more extensive discussion of our rectangles in terms of spatial frequency).

Furthermore, it appeared that the inserted line was either consistent or in conflict with the rectangle. Boer and Keuss (1981) found that classification was easier when the rectangle and the line were in the same state (both standing or both lying; see the left pair of stimuli in Figure 3) than when their states were in conflict (see the right pair of stimuli in Figure 3). The present Experiment 1 derives speedaccuracy functions separately for these conflicting and consistent rectangles.

\section{Method}

Subjects. Fourteen students took part in the experiment as paid volunteers. All had participated in the chronologically earlier Experiment 2.

Stimuli and Apparatus. The subject sat in a soundproof cubicle, with hands resting on a response box that had two conventional response keys mounted on it: the left key was to be used for reacting to lying stimuli, and the right key, to react to standing stimuli. The subject faced a black projection screen $140 \mathrm{~cm}$ away, its center slightly below eye level. The cubicle was dimly lit, giving the white outline of the otherwise black screen an illumination of $.4 \mathrm{~cd} / \mathrm{m}^{2}$. A feedback indicator lamp (showing whether the response was correct or not) was attached to the left of the screen, and a loudspeaker stood on the floor below the screen. All other equipment was outside the cubicle.

An electromechanical shutter (Compur $M$ ) was placed directly before the lens of a Kodak Carousel (S-RA 2000) that projected the stimuli. The shutter opened in less than $3 \mathrm{msec}$. The beam of the projector entered the cubicle through a $12 \times 12 \mathrm{~cm}$ window and showed the stimulus as a white outline rectangle on the black screen (white $=5.3$ and black $=.03 \mathrm{~cd} / \mathrm{m}^{2}$ ). The stimulus was a $2.52 \times 1.68 \mathrm{~cm}$ rectangle, subtending a visual angle slightly over $1 \mathrm{deg}$. Line thickness was about $2 \mathrm{~mm}$.

The to-be-judged aspect of the stimulus was the position-either standing or lying. The to-be-ignored aspect was the position of an inserted line that was also either standing or lying. Figure 3 shows the set of four possible stimuli.

Procedure. A trial had a fixed duration of $4.25 \mathrm{sec}$ and began with a 150 -msec warning $(75 \mathrm{~dB}, 1,000 \mathrm{~Hz})$ delivered by the loudspeaker below the screen. This was followed by the to-be-judged stimulus that was projected for $100 \mathrm{msec}$. When the deadline had elapsed, the response signal sounded, indicating that the subject should respond immediately by pressing one of the two response keys. The trial ended with a silent period of $3 \mathrm{sec}$, during which the projector searched for the next stimulus slide.

A deadline was defined as the time between stimulus onset and the response signal. The latter was a train of three pips, spaced by $75 \mathrm{msec}$, much as in the study of Schouten and Bekker (1967). Each pip lasted $20 \mathrm{msec}$ and had a frequency of $2,500 \mathrm{~Hz}$. The subject's instruction was to press one of the keys at the onset of the last pip. When he pressed the key more than $50 \mathrm{msec}$ too early or too late, a buzzer sounded for $200 \mathrm{msec}(61 \mathrm{~Hz}$, square wave). The subject was instructed to aim at the buzzer-free period of $100 \mathrm{msec}$. (For the experimental sessions, an average of $77 \%$ of the reactions were within the desired time-band.)

Time between warning tone and last pip was always $650 \mathrm{msec}$ (offset to onset), while the moment of stimulus presentation varied as required by the deadline. Time elapsed since warning tone was therefore an additional cue for the subject when the response was due. The interval between warning tone and stimulus presentation was, of course, always $650 \mathrm{msec}$ minus the deadline (msec).

A feedback indicator lamp lit a $2 \times 4 \mathrm{~cm}$ area during the time a key was down. A green light indicated a correct response, and a red light indicated an incorrect response. Deadlines were presented in blocks of 120 trials each. Deadlines were chosen in such a way that correct stimulus judgments for the individual subject should not be under $60 \%$ or over $95 \%$. Selection of deadlines was random with this constraint.

After a 120-trial block, the performance was discussed with the subject on the basis of a computer printout indicating percentage responses made too early or too late and percentage of correct responses.

Data from the first session were considered as practice. In the second session, scheduled on another day, the experimental data were collected.

\section{Results and Discussion}

Only the last eight blocks were entered in the analysis. Blocks with less than $55 \%$ correct responses were discarded (two blocks over all subjects). For each 110-trial block (first 10 trials discarded as warm-ups), two RT-accuracy points were derived: one for consistent stimuli and the other for conflict stimuli (see Figure 3). Garner's (1962) information transmission index, $U$, was used as a measure of accuracy. For the present two-choice task, this $U$ ranges between zero and one bit of transmitted information. Median reaction time was used as a measure of speed. The last eight blocks for each subject were thereby reduced to a group of eight RT-accuracy points for consistent stimuli, and another eight RT-accuracy points, for conflict stimuli. Points with accuracy under .01 or over .94 bits were discarded. This resulted in an average loss of .4 points per subject; the speed-accuracy function was thus estimated on an average of 7.6 points.

The SAF was calculated as the line best fitting the above-mentioned data. In view of the fact that both the RT and accuracy variables are subject to error (cf. Salthouse, 1981), Brace's method for best-fitting lines was adopted (Brace, 1977). Using this method, two speed-accuracy functions were derived for each individual subject: one for consistent, the other for conflict, stimuli.

To test whether the consistent SAF differed from the conflict SAF, two ANOVAs were employed. One ANOVA tested for slope differences; the other tested for intercept differences. In order to provide a more meaningful interpretation for the intercept, the latter was defined as the point of intersection with the abscissa, which can be interpreted as the moment that accuracy begins to improve from chance level. 
The slope ANOVA revealed only a trend toward faster information transmission for consistent stimuli $[F(1,13)=3.28, p<.10]$. The intercept ANOVA showed a significant effect of stimulus type: the moment that information transmission started occurred earlier for consistent stimuli [239 vs. $252 \mathrm{msec}$; $F(1,13)=11.05, p<.01]$. Figure 4 shows the SAFs, with slope and intercept averaged over the 14 subjects.

The global precedence prediction-no difference due to details in the initial part of the SAF-was disconfirmed. In the absence of a clear difference in slope, the two SAFs are best considered as parallel lines, with the entire conflict SAF displaced somewhat to the right (or slower/inaccurate range). This means that information about stimulus detail had an approximately equal effect over the whole speed-accuracy range studied. Anyway, there was no clear indication that the effect of irrelevant stimulus detail was increased from initial to later stages, as predicted by the idea of global precedence. The data suggested, rather, that local features had already been sampled by the time information about global features could be passed on. In that case, the slope of the SAF would vary only under the influence of postperceptual processes and would thus fail to reveal a differential time course of global and local features. However, it is possible that the differential time course might show as an intercept effect, if it is

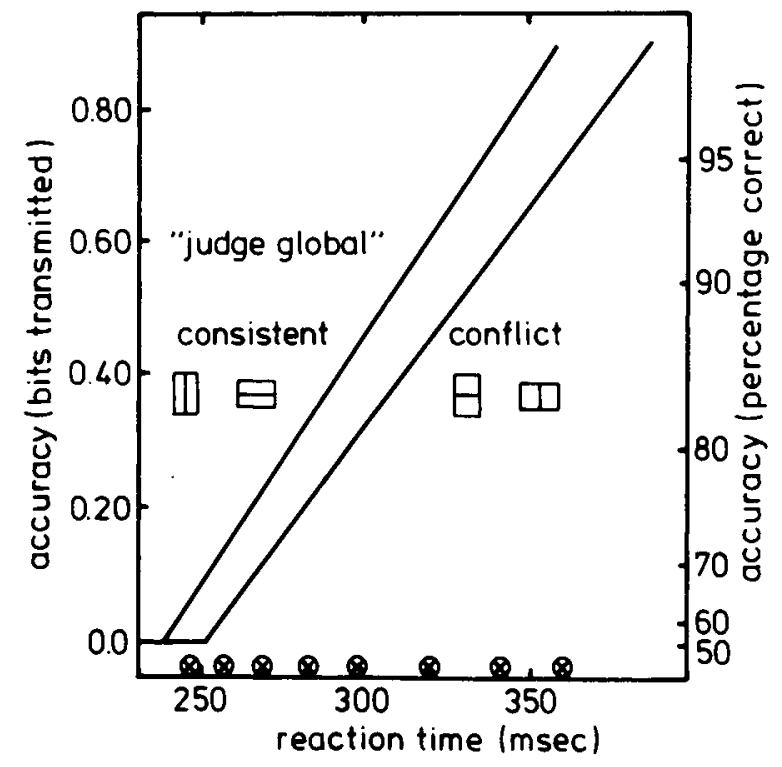

Figure 4. Speed-accuracy functions for the geometrical stim. uli used in Experiment 1 for each stimulus type (consistent/conflict). Accuracy is expressed as bits of information transmitted. To illustrate the relation with percentage correct reactions, percentage correct is shown on the right-hand scale of the figure. This relationship holds only when percentage correct is distributed evenly over stimuli, since uneven distribution raises the bits transmitted for a given percentage correct. (The eight symbols along the abscissa show the average deadlines used in the eight experimental blocks.) correct to suppose that the start of information transmission occurs as soon as sufficient features have been sampled. One of the aims of Experiment 2 was to see whether global precedence was indeed reflected in the intercept of the speed-accuracy functions.

\section{EXPERIMENT 2}

The stimuli of Experiment 1 are not very typical in studies of global precedence. To avoid the possibility that the geometric stimuli failed to capture the global-local distinction, the letter stimuli of Figure 1 were used in Experiment 2. All research of global precedence has so far used this type of material, in which many small letters constitute the global form (Hoffman, 1980; Martin, 1979; Miller, 1981a; Navon, 1977, 1981a).

In order to test the global precedence model further, the experiment was extended to include a judgeglobal group of subjects as well as a judge-local group. The global precedence model predicts that the moment at which information transmission can start will occur later in time for the judge-local group, because local features are sampled later, or, in a weaker version of the model, because the rate of sampling local features is relatively slow. The moment at which sufficient features have been sampled for a betterthan-chance impression of the stimulus will consequently be later.

A further prediction of the model is that global precedence will be obtained from the beginning of the SAF, observed as a sizable interference when global features are in conflict with local judgment, compared with a minor interference when local features are in conflict with global judgment. The weak form of global precedence implies that the momentary sample contains more global than local features during the initial stages. It follows that conflict in the stimulus (top-right and bottom-left in Figure 1) will be most interfering when the local features, assumed to be underrepresented, have to be judged, compared with when the overrepresented global features have to be judged.

In addition to these points, we examined the role of stimulus-response compatibility. Navon (1977, 1981a) did not analyze this factor, although it has some theoretical importance: interaction between the global precedence result and S-R compatibility would suggest that global precedence was caused at a postperceptual stage-namely, at response selection. This is especially relevant because of Miller's (1981a) contention that a global precedence result has a postperceptual locus.

In these related studies, the stimulus could appear in any one of the four quadrants of an area about twice the size of the global form. Although not explicitly stated, the likely reason for introducing this positional uncertainty is to prevent the subject from 
limiting his attention to a particular spot-a strategy that would be very useful, especially in the judgelocal condition. (However, Ward, in press, compared positional certainty and uncertainty and found no difference whatever.) Martin (1979) also randomized stimulus position, but, due to the fact that her subjects named the stimuli, there were no consequences for S-R compatibility. We, like Navon $(1977,1981$ a), used two different response keys: one on the left and one on the right. It is in this particular situation that a left-right variation in stimulus position works as an irrelevant directional cue for responding with the left vs. the right key. Simon has studied the effect extensively and has shown that this compatibility is located at the response selection stage (Simon, in press; Simon, Acosta, Mewaldt, \& Speidel, 1976). Applying Sternberg's (1969) additive-factor logic, interaction of Factor $x$ with S-R compatibility implies that Factor $\mathrm{x}$ has its effect also at the response selection stage. The logic thus enables us to see whether global precedence is (in part) located at this postperceptual stage.

\section{Method}

Subjects. Thirty-four students were tested; all were paid volunteers with normal or fully corrected vision. Seventeen subjects were assigned to each of the two attention conditions-judge local and judge global. However, two judge-global subjects and one judge-local subject had to be eliminated from the final analysis since their performance deteriorated during the first session, while accuracy was hardly related to the particular deadline. Discarding randomly another subject from the judge-local group made for a balanced design of 15 judge-global and 15 judge-local subjects.

Stimuli. The stimuli, their positions on the display, exposure time, and visual angle were very much the same as the manyelement stimuli in Martin's (1979) first experiment. The global letter was $9.9 \times 6.3 \mathrm{~cm}$ or $2.6 \times 4.0$ deg of visual angle at a $140-\mathrm{cm}$ viewing distance. The local letters were $8.5 \times 11.2 \mathrm{~mm}$, which is the line thickness of the global letter; line thickness of the local letters was less than $2 \mathrm{~mm}$. The stimulus appeared randomly in one of the four quadrants of the visible area immediately adjacent to the area's central and vertical axes. Thus, half of the stimuli were presented on the left side of the screen (a quarter at top left, a quarter at bottom left), and half of the stimuli were presented on the right side of the screen (a quarter at top right, a quarter at bottom right). Half of the stimuli required a compatible reaction (stimulus left, response left; stimulus right, response right), and half required an incompatible reaction. Furthermore, in addition to the consistent and conflict stimuli, a neutral type was used in which the irrelevant level was a rectangle not connected to any to-be-judged aspect (see Figure 1).

Procedure. The procedure was the same as in Experiment 1.

\section{Results}

Data analysis was similar to Experiment 1, but compatibility was also analyzed. For a particular subject, six speed-accuracy functions were derived (3 stimulus types $\times 2$ levels of compatibility), based on RT-accuracy points that were between .01 and .94 bits of information transmitted. (Due to the greater number of SAFs and the consequently lower number of observations per point, the criterion was in fact between .01 and .76 bits.) The criterion re-

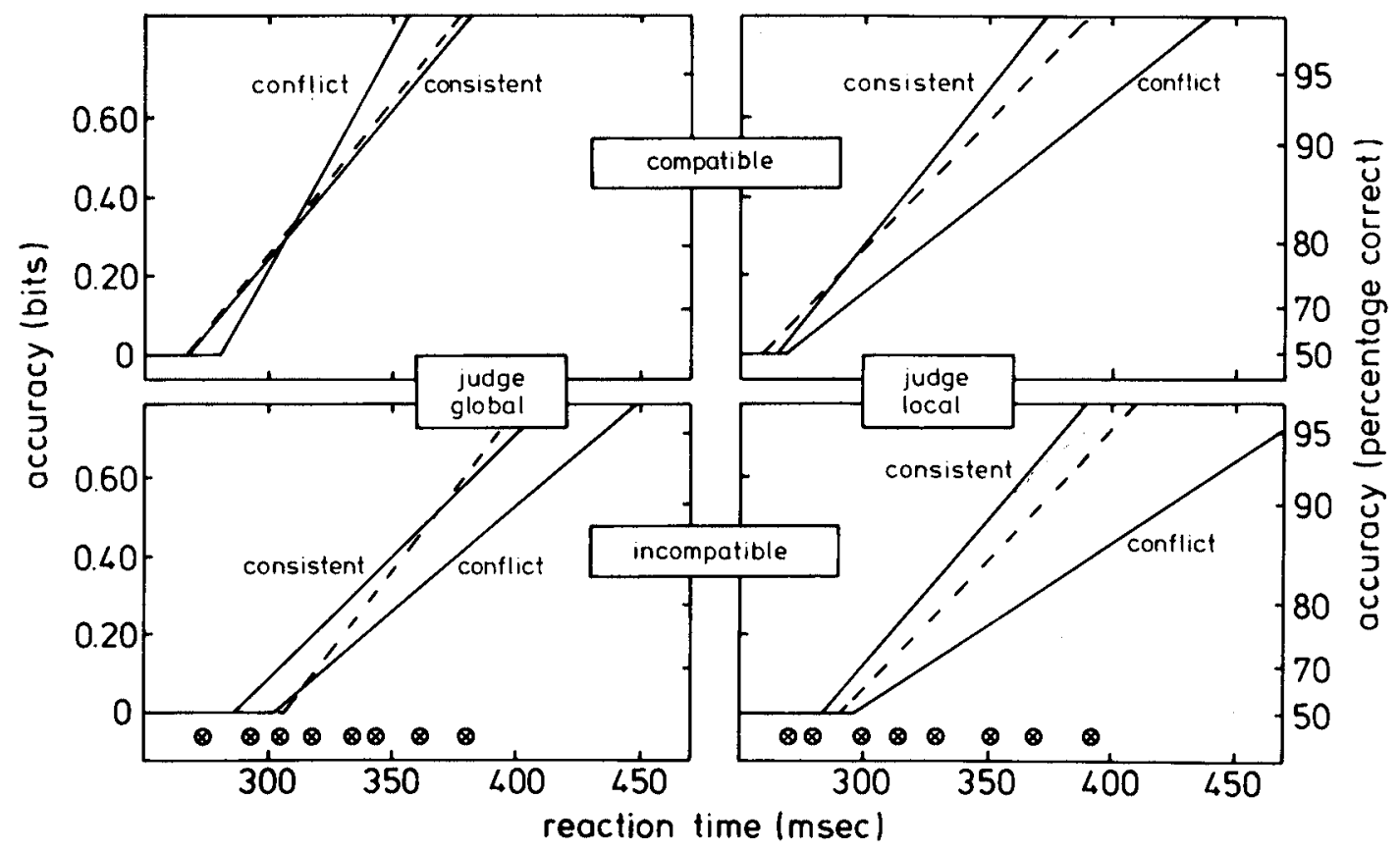

Figure 5. Speed-accuracy functions for the letter stimuli used in Experiment 2 for each stimulus type: the broken line represents neutral stimuli; the solid lines show consistent and conflict stimuli, as indicated. The eight symbols along the abscissa show the average deadlines used. (The left part of the figure shows the data of the 15 judge-global subjects; the right part shows the data for the 15 judge-local subjects. Each top panel shows data for which the stimulus required a compatible reaction; each bottom panel shows data for which the stimulus required an incompatible reaction.) 
moved, on the average, 1.5 points per subject. Each SAF was thus based on 6.5 points.

Figure 5 shows the average SAFs for each stimulus type. The left part of the figure shows the judgeglobal group of subjects; the right part shows the judge-local group of subjects. The top panel within each part shows the compatible condition, and the bottom panel shows the incompatible condition.

Compatibility had an effect on the slope $[F(1,28)$ $=4.59, \mathrm{p}<.05]$ and on the intercept $[F(1,28)=51$, $\mathrm{p}<.01]$; that is, incompatible $\mathrm{S}-\mathrm{R}$ mappings delay by $24 \mathrm{msec}$ the moment that information transmission is begun and subsequently slow down the transmission itself so as to predict another 24-msec delay under perfect accuracy performance. Compatibility did not interact with other factors except for a trend toward interaction with stimulus type $[\mathrm{Fs}(2,56)=$ 2.30 and 2.49 , ps $<.11$; for slopes and intercepts, respectively].

Stimulus type tended to affect the intercept $[F(2,56)$ $=3.03, \mathrm{p}<.06]$, pointing to the finding that transmission may start a little earlier for consistent stimuli (but see also the General Discussion). Global subjects tended toward greater speed of information transmission than local subjects $[F(1,28)=3.68, p<.07]$, while information transmission started at the same time for both groups of subjects $(F<1)$. More important, the interfering effect of stimulus type on speed of information transmission (i.e., the slope of the SAF) was greater for the judge-local group $[F(2,56)$ $=5.34, \mathrm{p}<.01]$. No other interactions with global/ local subjects were observed.

On inspection of Figure 5, it is clear that the bulk of the effects, trends, and interactions related to stimulus type were accounted for by the shallow conflict SAF in the judge-global group. Information transmission was apparently very difficult in this condition.

\section{GENERAL DISCUSSION}

The typical global precedence result was obtained, insofar as the speed of information transmission was slowed down only when global features interfered with local judgment, and not when local features interfered with global judgment. Closer inspection of the data suggested a postperceptual basis for the results. The argument is mainly based on how the details interfered when the subject judged the stimuli on global form.

The first experiment revealed an effect of irrelevant local detail on the intercept of the SAF, while no clear evidence was observed for a slope difference. The second experiment confirmed the impression: the intercept effect-even the size of it-was replicated [13 msec; $F(2,14)=4.68, p<.05 ; F<1$ for the judgelocal group], while irrelevant local features had absolutely no effect on slope. Consequently, the effect of to-be-ignored detail must have been present in full from the very beginning of the SAF, while the effect was not further increased in the late part of the SAF. Thus, it is very likely that global and local features had already been sampled exhaustively before any information could be passed on. This was also suggested by the observation that information transmission for the judge-local group started just as early as for the judge-global group; in fact, it was $10 \mathrm{msec}$ earlier.

We now consider when global precedence was obtained. No global precedence was found at the intercept; the different SAFs for different stimulus types lie very closely together, to diverge substantially only in the late part of the SAF. If perception (or feature accumulation) was already complete at the intercept, the absence of an initial global precedence result argues against any perceptual locus for global precedence. When, later on, a global precedence result was obtained, its locus was consequently postperceptual. Miller (1981a) similarly argues for postperceptual global precedence. He showed that local information becomes available to decision processes with a time course similar to that of global information and that subjects can integrate global and local information into a single decision. His conclusion may have been dependent on the specific task: he used a target-search in which the target could be located at either or both levels. This paradigm more or less forced the subject to divide his attention over global and local aspects. The present task, by contrast, motivated the subject to lock his attention onto one aspect exclusively. (Moreover, we used a betweensubjects design.) Our result is thus an even stronger argument in favor of similar time courses for the availability of local and global aspects in perception.

The evidence for similar time courses in perception does not question the phenomenon itself-namely, that it is impossible to ignore the global aspect. The phenomenon was observed in this and other studies and appears to be mandatory, in the sense that subjects were unable to ignore the global aspect in spite of their intentions. It is only the interpretation in terms of perception that is probably incorrect. Global precedence is a matter of differential use of global and local information, not a matter of differential availability of global and local information (see the discussion between Miller, 1981b, and Navon, 1981b). The term global dominance may be preferred, because it avoids the implication of differential availability.

In fact, when it comes to perceptual availability, the data of the present paper are even compatible with the opposite building order of the percept: local before global or, at least, local as the faster information. If we are right in interpreting the intercept as reflecting an early stage of perception determined by data limitations rather than processing limitations, the intercept data reveal that the local, and not the global, aspect is most difficult to ignore. The irrelevant global aspect had no effect on the intercept for 
the judge-local group (Experiment 2), but the irrelevant local aspect had a significant effect on the intercept, both in Experiment 1 and in Experiment 2 (the judge-global group).

At what stage of processing does global dominance come into being? The position seems defendable that stimulus preprocessing and encoding were either very fast relative to subsequent stages or that they were unitary processes in the sense that they did not pass information to subsequent stages unless they were completed. ${ }^{3}$ There was no indication that the result of global dominance had something to do with response selection (Fs $<1$ for the interaction with S-R compatibility). The most tenable interpretation of the present results seems to be that global dominance (or precedence) is located somewhere between perception and response selection.

Miller (1981a) argues for greater ease of directing attention to the global aspect and deciding accordingly. Ward (in press) similarly invokes an attentional account when ascribing global dominance to the greater conspicuity of the global aspect because he leaves the precise definition of conspicuity to Engel (1971), who defines conspicuity as "that combination of properties in a visible object in its background by which it attracts attention" (p. 536). Unfortunately, attentional accounts are of little help in identifying which processing stages are involved in global dominance.

Finally, we briefly consider a finding of local precedence, which may suggest a condition necessary to obtain global dominance. The particular result was obtained in the card-sort tasks of Pomerantz and Sager (1975). The stimuli in their last two experiments were very similar to our letter stimuli: large forms $(2.5 \mathrm{deg})$ made up of many small letters $(.2 \mathrm{deg}$ each). They found that the local letters were most difficult to ignore.

The first consideration is the difference in methods used to assess the interference of the to-be-ignored aspect. The card-sort task investigates the effect of random variation in the irrelevant aspect compared with a condition without this irrelevant variation. The task in the present report investigates the effect of a particular state of the irrelevant aspect compared with the effect of other states of that aspect. For example, we were interested in the effect of conflict due to the irrelevant aspect compared with the effect of other states (neutral and consistent) of the aspect. In assessing such effects, we used trial blocks in which the state of the irrelevant aspect varied unpredictably from trial to trial, but we did not use trial blocks with the irrelevant aspect fixed in a particular state. There is, however, some counterevidence for the idea that the different result of Pomerantz and Sager (1975) was due to their different method. We previously examined the rectangular stimuli of Experiment 1 in a card-sort task and found a global dominance result.
Navon (1981a) also used the two methods (even in a within-subjects design) and also found global dominance in both methods.

The difference in stimuli suggests a more likely hypothesis to account for the absence of global dominance in the results of Pomerantz and Sager (1975)namely, that the relation between the value of the global aspect and the particular value of the local aspect is very important. Pomerantz and Sager choose letters for the local aspect and geometric configurations for the global aspect, thereby avoiding conflict across aspects. On the other hand, in those studies in which global dominance was found, the levels within a particular aspect were selected in such a way as to contradict or to confirm the levels selected for the other aspect. This suggests that global dominance is only observed when the levels across aspects are sometimes in conflict; in other words, that the consistent/conflict relation across aspects is a necessary condition for global dominance. The global aspect must have some bearing on the judgment the subject tries to make-only then is it impossible to ignore the suggestion offered by the global aspect.

\section{REFERENCES}

Boen, L. C., \& Keuss, P. J. G. Storage mode of geometrical dimensions in a two-stimulus matching paradigm. Perception \& Psychophysics, 1979, 25, 197-204.

Boen, L. C., \& Keuss, P. J. G. Integrality over different paradigms: (1) card sorting and (2) two-stimulus matching. Perception \& Psychophysics, 1981, 29, 71-76.

Bouma, H. Visual recognition of isolated lower-case letters. Vision Research, 1971, 11, 459-474.

Brace, R. A. Fitting straight lines to experimental data. American Journal of Physiology, 1977, 233, R94-R99.

Campelel, F. W. The transmission of spatial information through the visual system. In F. O. Schmitt \& F. G. Worden (Eds.), The neurosciences third study program. Cambridge, Mass: M.I.T. Press, 1974.

ENGEL, F. L. Visual conspicuity, directed attention and retinal locus. Vision Research, 1971, 11, 563-576.

Eriksen, C. W., \& Schultz, D. W. Temporal factors in visual information processing: A tutorial review. In J. Requin (Ed.), Attention and performance VII. Hillsdale, N.J: Erlbaum, 1978.

Eriksen, C. W., \& Schultz, D. W. Information processing in visual search: A continuous flow conception and experimental results. Perception \& Psychophysics, 1979, 25, 249-263.

GARNER, W. R. Uncertainty and structure as psychological concepts. New York: Wiley, 1962.

Gibson, E. J. Principles of perceptual learning and development. New York: Appleton, 1969.

Hoffman, J. E. Interaction between global and local levels of a form. Journal of Experimental Psychology: Human Perception and Performance, 1980, 6, 222-234.

KinCHLA, R. A., \& WOLFE, J. M. The order of visual processing: "Top-down," "bottom-up," or "middle-out." Perception \& Psychophysics, 1979, 25, 225-231.

LUPKER, S. J. On the nature of perceptual information during letter perception. Perception \& Psychophysics, 1979, 25, 303-312.

Martin, M. Local and global processing: The role of sparsity. Memory \& Cognition, 1979, 7, 476-484.

Millen, J. Global precedence in attention and decision. Journal of Experimental Psychology: Human Perception and Performance, 1981, 7, 1161-1174. (a) 
Mille R, J. Global precedence: Information availability or use? Reply to Navon. Journal of Experimental Psychology: Human Perception and Performance, 1981, 7, 1183-1185. (b)

Navon, D. Forest before trees: The precedence of global features in visual perception. Cognitive Psychology, 1977, 9, 353-383.

Navon, D. The forest revisited: More on global precedence. Psychological Research, 1981, 43, 1-32. (a)

NAvon, D. Do attention and decision follow perception? Comment on Miller. Journal of Experimental Psychology: Human Perception and Performance, 1981, 7, 1175-1182. (b)

Pachella, R. G. The interpretation of reaction time in informationprocessing research. In B. H. Kantowitz (Ed.), Human information processing: Tutorials in performance and cognition. Hillsdale, N.J: Erlbaum, 1974.

Petersik, J. T. A physiological and psychophysical interpretation of geometrical dimensions in a binary classification task: Comments on Keuss (1977). Perception \& Psychophysics, 1977, 22, 511-515.

PoLF, J. The word superiority effect: $A$ speed accuracy analysis and test of a decoding hypothesis. Unpublished doctoral cissertation, University of Oregon, 1976.

Pomerantz, J. R., \& Sager, L. C. Asymmetric integrality with dimensions of visual pattern. Perception \& Psychophysics, $1975,18,460-466$.

Salthouse, T. A. Converging evidence for information-processing stages: A comparative-influence stage-analysis method. Acta Psychologica, 1981, 47, 39-61.

Schouten, J. F., \& Bekken, J. A. M. Reaction time and accuracy. In A. F. Sanders (Ed.), Attention and performance $l$. Amsterdam: North-Holland, 1967. (Reprinted from Acta Psychologica, 1967, 27.)

Selfridge, O. Pandemonium: A paradigm for learning. In Symposium on the mechanization of thought processes. London: Her Majesty's Stationery Office, 1959.

Simon, J. R. Effect of an auditory stimulus on the processing of a visual stimulus under single- and dual-tasks conditions. Acta Psychologica, in press.

Simon, J. R., Acosta, E., Mewaldt, S. P., \& Speidel, C. R. The effect of an irrelevant directional cue on choice reaction time: Duration of the phenomenon and its relation to stages of processing. Perception \& Psychophysics, 1976, 19, 16-22.

Sternberg, S. The discovery of processing stages: Extensions of Donders' method. In W. G. Koster (Ed.), Attention and performance II. Amsterdam: North-Holland, 1969. (Reprinted from Acta Psychologica, 1969, 30.)

Treisman, A. M., \& Gelade, G. A feature-integration theory of attention. Cognitive Psychology, 1980, 12, 97-136.

VARTABEDIAN, A. G. Legibility of symbols on CRT displays. Applied Ergonomics, 1971, 2, 130-132.

WARD, L. M. Determinants of attention to local and global features of visual forms. Journal of Experimental Psychology: Human Perception and Performance, in press.

\section{NOTES}

1. According to a reviewer of the present paper, the plotting order in Hoffman's second experiment was random. It was not made clear whether this refers to the order of plotting the small letters or to the order of plotting each point over the whole stimulus. Only in the latter case is our objection met, but see our incidental finding that brightness differences due to oscilloscope presentation can override global precedence.

2. The data of the left and right panels of Figure 2 were based on one group of 18 , and another group of 11 , subjects, respectively, none of whom participated in the other experiments reported in this research. Each subject did 150-200 judge-local trials and 150-200 judge-global trials, with order of global-local attention counterbalanced between subjects.

3. The result that the slope of the speed-accuracy function does not reflect perceptual processes argues against the applicability of the continuous-flow model of Eriksen and Schultz $(1978,1979)$. The result is in line with Pachella's (1974) idea that the slope reflects only process limitations-not data limitations.

(Manuscript received December 30, 1981; revision accepted for publication January 14, 1982.) 